\title{
Research on Eco-geological Environment Carrying Capacity for Pingwu County after Wenchuan Earthquake Based on Modified AHP
}

\author{
pu wang \\ Tianjin University https://orcid.org/0000-0002-7945-5109 \\ Yimin Liu ( $153973418 @ q q . c o m$ ) \\ Tianjin University of Technology
}

\section{Research Article}

Keywords: Eco-geological Environment Carrying Capacity, Modified AHP, Evolutionary Algorithms, Wenchuan Earthquake Posted Date: August 13th, 2021

DOI: https://doi.org/10.21203/rs.3.rs-731470/v1

License: (c) (i) This work is licensed under a Creative Commons Attribution 4.0 International License. Read Full License 


\section{Abstract}

Many counties in southwestern China are devastated by the Wenchuan earthquake, and the earthquake also induced lots of geological hazards. So evaluation of the eco-geological environment in the disaster area is essential for county restoration and ecological remediation. This research accounts for Pingwu County, which is $89 \mathrm{~km}$ to the epicenter of the Wenchuan earthquake, as a study area to evaluate eco-geological environment carrying capacity. Firstly, three evaluation index layers are built to the eco-geological environment carrying capacity model after the earthquake, including geological environment, ecological environment, and social environment. And then, the evolutionary algorithm(EA) is used to modify Analytic Hierarchy Process(AHP). Through optimizing consistency ratio of AHP, the best consistent evaluation matrix can be obtained. Finally, We calculate the eco-geological environment carrying capacity of Pingwu by the best consistent evaluation matrix, and obtain hierarchical map of the eco-geological environment carrying capacity through grid rasterization. The results show that current eco-geological environment carrying capacity in Pingwu county mainly contains two states, equilibrium and surplus. According to the hierarchical map, this study suggests optimizing the construction of the central area in Pingwu, controlling the scale of regional construction, and maintaining the original ecological species in the natural protection area.

\section{Introduction}

A strong earthquake like the Ms8.0 Wenchuan earthquake leads to catastrophic disasters in most counties of southwest China(Wang et al. 2011; Ye et al. 2011). At the same time, landslides, barrier lakes, and collapses induced by the earthquake have seriously threatened the stable development of the social economy and ecological environment in the disaster area(Zhang et al. 2010). The Fujiang river basin belongs to the severely afflicted area of the Ms7.2 Songping Earthquake in 1976 and the Ms8.0 Wenchuan Earthquake in 2008. The eco-geological environment of the basin is complicated and fragile. As a result, there are a large number of secondary disasters in the area after the earthquake. Pingwu County, one of the key towns in the Fujiang river basin, is being rebuilt after the earthquake. With the increase of human engineering activities in reconstruction, the geological environment capacity of some regions is rapidly decreasing and not adapt to further construction and development. Therefore, it is essential to implement eco-geological environmental carrying capacity assessment in the area to plan the town's development and provide chronic guidance for urban reconstruction in the disaster area.

The capacity of the geological environment for human activities is limited, and the maximum limit is the geological environment capacity(Bishop 1974). Since this concept was put forward, the evaluation method of the carrying capacity of the geological environment has been improved. Rees and Wackernagel (1992)proposed the ecological footprint method by measuring the ecological environment range required by human activities, it provides a basis for judging whether human activities are within the adaptable range of carrying capacity. Marco et al. (2000) carried out a multi-index evaluation system to evaulate the geological environment carrying capacity in, the indexes including water/soil pollution, land subsidence, land salinization, and desertification. Cherp (2001) and Arild (2009) used systematic analysis methods to evaluate and compare the geological environment carrying capacity of countries located in Eastern Europe and Central Asia. They summarized the indexes and methods of evaluation for the geological environment carrying capacity. In recent years, the GIS-based analytic hierarchy process is applied to the evaluation of geological environment carrying capacity. Zhu (2009) takes Guangxi Beibu Gulf Economic Zone as the research object, using the AHP with comprehensive index method and the coupling method of environmental saturation to make early warnings of the resource and environmental constraints, and formulated prevention and control measures. Wang et al. (2015) used the AHP-GIS coupling model to evaluate the carrying capacity of the geological environment in the Shandong Peninsula. However, After the Wenchuan earthquake, few research studies combine the different eco-geological environment factors of key towns in the disaster area, lack of guidance for long-term eco-geological recovery from the view of eco-geological environment carrying capacity. 
In this study, the following aspects will be conducted for evaluating eco-geological environment carrying capacity in Pingwu: (1) The AHP method is modified by using the evolutionary algorithm, which solves the problem that the judgment of experts scoring lead to the AHP method is not objective. (2) Through the geological survey of the geological structure and natural hazards, we determine the ten factors related to the geological environment, ecological environment, and social environment. The current status of the geological environment bearing capacity in Pingwu County is calculated by the modified AHP method. (3) According to the hierarchical map, we analyze the geological environment carrying capacity threshold and capacity status of Pingwu county and provide a development suggestion for the post-earthquake development plan of the region.

\section{Geological Environmental Background Of The Study Area}

The study area, Pingwu County, is located in the northwestern part of the Sichuan Basin, within the eastern edge of the transition from the Qinghai-Tibet Plateau to the Sichuan Basin(E103 ${ }^{\circ} 50^{\prime} \sim \mathrm{E} 104^{\circ} 58^{\prime}, \mathrm{N} 31^{\circ} 59^{\prime} \sim \mathrm{N}^{\circ} 3^{\circ} 02^{\prime}$, with a total area of $5974 \mathrm{~km}^{2}$ ). The geographic location and elevation information map of Pingwu County is shown in Fig. 1. The overall terrain of the study area is high in the northwest and low in the southeast. The altitude of Xuebaoding, the highest peak of Minshan Mountain in the northwest up to 5588 meters, and the lowest in the southeast is Jiaoyuanzi Valley of Erlangxia just 600 meters, Fujiang River. The height difference between the two places is nearly 5000 meters.

As shown in Fig. 1, Pingwu County is $89.7 \mathrm{~km}$ from the epicenter of the Ms8.0 Wenchuan earthquake, which belongs to the high-hazard area within the epicenter, and the disasters in towns near Beichuan County are more serious than other areas. The 2008 earthquake sequence is distributed along the Wenchuan-Maowen fault, and half of the earthquakes passed through Pingwu. Most of the focal mechanism solution is thrust, and others are strike-slip. At present, there are still many geological environment problems in Pingwu County, including the following aspects: Firstly, Soil erosion is widely happened in this area due to earthquakes and human engineering activities, especially after the Wenchuan earthquake. Secondly, Pingwu county belongs to the mountainous area, and mountain valley catchment capacity leads to flood disasters more serious. There are many collapse deposits in the valley after the earthquake, blocking the channel, resulting in poor drainage and prone to flood in heavy rainfall. And then, The foundation of the Wuxian structure is placed on the soil foundation with different physical and mechanical properties, which is prone to uneven settlement. Last and most important, there are several active faults in the northwest triangle block of Pingwu, including Minjiang fault, Huya fault, Songpinggou fault, and Jiaochang fault, and this area has happened twenty Ms > 5 earthquakes and three Ms > 7 earthquakes since 1630 .

On May 12, 2008, Ms 8.0 magnitude earthquake occurred in Longmenshan fault. The Pingtong-Xingyan-Nanba-Shikan area along the fault in Pingwu County is meizoseismal area, and seismic intensity is as high as 11 degrees. A total of 27 aftershocks with Ms > 4 occurred in Pingwu county and adjacent areas, the maximum magnitude of aftershocks in this area up to Ms 6.1. Therefore, a great deal of secondary geological disasters is induced by the earthquake. According to the detailed investigation of geological disasters(Qin et al. 2009), there are 406 geological hazards in Pingwu County. As shown in Fig. 2, Landslides account for the highest proportion of disasters, reaching $64.53 \%$ (262 sites). The second is debris flow, which develops 66 sites and accounts for $16.26 \%$ of all. Others include 47 collapse sites (11.58 \%) and 31 unstable slopes sites(7.64\%). The threat of geological disasters mainly includes towns, settlements, schools, farmer's lands, and roads.

\section{Evaluation Of Eco-geological Environment Carrying Capacity In Pingwu County 3.1 A modified AHP by evolutionary algorithms}

It is the first time that Satty et al. (Saaty and Kearns 1985)proposed a multicriteria decision-making method named Analytic Hierarchy Process(AHP), which is considered to be a reasonable and effective method of determining power in the existing 
weight evaluation methods(Saaty 2008). Because AHP adopts the qualitative analysis of the expert scoring method and the appropriate mathematical model for quantitative analysis, multiple objectives and multiple criteria can be reasonably quantitatively analyzed simultaneously. (Saaty and Vargas 2012) The way includes four main steps: 1.Establish the analytic hierarchy model. 2. Building model comparison matrix. 3. Calculate the weight of each factor in the model. 4. Results consistency test. However, the subjective influence of expert evaluation in AHP leads to the qualitative aspects too many ingredients and reduces the reliability of this method(Zhao J and Dan Q 2003). This study uses evolutionary algorithms(EA) further to optimize the weight determination process of AHP and improve the rationality of the quantitative evaluation of each factor.

As described in Figure 3, the modified AHP uses evolutionary algorithms to calculate the optimal compare matrix with the best consistency(the black dotted line), instead of expert scoring and checking consistency in the traditional AHP (the red dotted line). As a result, the modified AHP method is more objective than the evaluation result of the traditional method.

Through the improvement of traditional AHP methods, the step of the modified AHP is organized as follows. Firstly, determining the evaluation indicators $(X 1, X 2, \ldots, X n)$ of judgment matrix $X$. And then, obtaining the importance range by pairwise comparison of indicators refer to Table 1. At last, the fuzzy judgment matrix is assembled by the importance range. It is worthy to note that the importance of the two evaluation indicators is between two scales, so we construct the fuzzy scoring matrix by the expert scoring interval and determine the comparison matrix with the optimal consistency within the scope of the fuzzy scoring matrix.

Table 1 The standard for quantitative judgment of importance for index

\begin{tabular}{|ll|}
\hline Scale & Meaning \\
\hline 1 & Indicating that two factors are equally important \\
\hline 3 & Indicating that one factor is slightly more important than the other \\
\hline 7 & Indicating that one factor is significantly more important than the other \\
\hline 9 & Indicating that one factor is more important than the other \\
\hline $2 \varangle 4 \varangle 6 \otimes 8$ & Indicating that one factor is extremely more important than the other \\
\hline Reciprocal & $\begin{array}{l}\text { The factor } i \text { is compared with } j \text { to obtain the judgment } b_{i j} \text {. The factor } j \text { is compared with } i \text { to obtain the } \\
\text { judgment } \mathrm{b}_{\mathrm{ji}}=1 / \mathrm{b}_{\mathrm{ij} \text {. }}\end{array}$ \\
\hline
\end{tabular}

The borders of the evolutionary algorithm are defined by fuzzy judgment matrix, the same as traditional AHP, consistency ratio $C R$ calculated by equation 1-4 as the single optimization objective of EA.

बExpand the judgment matrix by row:

$$
V_{i}=\sqrt[n]{\prod_{j--1}^{n} x_{i j}} \quad(i=1,2 \ldots, \mathrm{n})
$$

『Normalized judgment matrix:

$$
W_{i}=V_{i} / \sum_{k=1}^{n} V_{k} \quad(i=1,2 \ldots, \mathrm{n})
$$

$\varangle$ Solving the maximum eigenvalue of the judgment matrix: 
$\lambda_{\max }=\frac{1}{n} \sum_{i=1}^{n} \frac{(X 1)_{i}}{a_{i}} \quad(i=1,2 \ldots, \mathrm{n})$

$\bowtie$ Calculating the consistency indicator $C R$ :

$$
\begin{aligned}
& C I=\left(\lambda_{\max }-n\right) /(n-1) \\
& C R=C I / R I
\end{aligned}
$$

$\mathrm{Cl}$ is the consistency ratio, $\lambda_{\max }$ is the maximum eigenvalue, and $\mathrm{n}$ is the matrix order. $R /$ is the ratio of average random consistency to random consistency reflected in the number of evaluation indicators. The judgment matrix would be ragard as satisfactory consistency, and the weight values are reasonable if $C R<0.1$ in the traditional method. However, If $C R>0.10$, the data will not generate meaningful outcomes unless reexamined, which always leads the evaluation procedure complicated. So we could take $C R$ as the only optimization objective to find a consistent optimal matrix as a single objective evolutionary problem, and directly calculate the consistent optimal judgment matrix by evolutionary algorithms method. So the result of modified AHP will be more objective than traditional way.

\subsection{Buliding the evaluation index system}

According to the post-earthquake geological environment background, ecological environment, distribution of disaster points, and social development in the region, the evaluation system of geological environment carrying capacity is constructed based on ten evaluation index layers in three aspects of geological environment, ecological environment, and social environment. Then the content of the evaluation index system of geological environment carrying capacity is calculated at different levels. Pingwu county geological environment carrying capacity evaluation index system structure, as shown in Figure 4, includes three subsystems: geological environment subsystem, ecological environment subsystem, and social-economic subsystem.

As can be seen in Figure 4, the prerequisite of building AHP evaluation system requires that the selected factors are independent of each other. Therefore, we select four factors related to geological environment carrying capacity for the geological environment criterion layer. Because the ecological environment is an essential part of environmental geology, the ecosystem is regarded as the second criterion layer, including water resources and tourism resources. The third criterion

layer is social economy, including population and basic facilities. Obviously, it is not comprehensive enough that evaluating regional geological environment carrying capacity just considering the potential of natural hazards and environmental resources(Liu et al. 2009; Wang and Yi 2009). The social environment and ecological environment are also key criterion layers in the quantitative model of eco-geological environment carrying capacity evaluation system. Therefore, we could comprehensively evaluate eco-geological environment carrying capacity of study area based on this new evaluation index system.

\subsection{Calculating weight of index by evolutionary algorithms}

According to the evaluation indexes of eco-geological environment carrying capacity in the last section and standard for quantitative judgment in Table 1, we determine the fuzzy judgment matrix referring to the expert scoring of AHP evaluation index in previous studies(Chen et al. 2004; Wang and Yi 2009). The fuzzy scoring matrix reflects the advantages of quantitative evaluation of expert scoring and reduces the objectivity caused by human factors in the scoring process. Table 2 shows the details about the fuzzy judgment matrix.

Table 2 Fuzzy judgment matrix of evaluation indexs 


\begin{tabular}{|c|c|c|c|c|c|c|c|c|}
\hline Index layer & $\begin{array}{l}\text { Geological } \\
\text { Structure }\end{array}$ & Lithology & Topography & $\begin{array}{l}\text { Natural } \\
\text { Hazard }\end{array}$ & $\begin{array}{l}\text { Water } \\
\text { Resources }\end{array}$ & $\begin{array}{l}\text { Mine } \\
\text { Resources }\end{array}$ & Population & $\begin{array}{l}\text { Basic } \\
\text { Facilities }\end{array}$ \\
\hline $\begin{array}{l}\text { Geological } \\
\text { Structure }\end{array}$ & 1 & $0-1$ & $0-1$ & $0-1$ & $4-5$ & $0-1$ & $0-1$ & $0-1$ \\
\hline Lithology & & 1 & $0-1$ & $0-1$ & $4-5$ & $0-1$ & $0-1$ & $0-1$ \\
\hline Topography & & & 1 & $0.5-1.5$ & $4-5$ & $0-1$ & $0-1$ & $0-1$ \\
\hline $\begin{array}{l}\text { Natural } \\
\text { Hazard }\end{array}$ & & & & 1 & $4-5$ & $0-1$ & $0.5-1.5$ & $0-1$ \\
\hline $\begin{array}{l}\text { Water } \\
\text { Resources }\end{array}$ & & & & & 1 & $0-1$ & $0-0.5$ & $0-0.5$ \\
\hline $\begin{array}{l}\text { Mine } \\
\text { Resources }\end{array}$ & & & & & & 1 & $0-0.5$ & $0-0.5$ \\
\hline Population & & & & & & & 1 & $1-2$ \\
\hline $\begin{array}{l}\text { Basic } \\
\text { Facilities }\end{array}$ & & & & & & & & 1 \\
\hline
\end{tabular}

In fact, it is a single-objective optimization problem of a complex process to select the consistent optimal matrix in the fuzzy matrix. $C R$ is the optimization objective of evolutionary algorithms in this study. We set the number of individuals to 1000 and increase the generations of evolution step by step. As shown in Fig. 5, With the increase of evolutionary generations, the mean and standard deviation of $C R$ in the last generation generations decrease continuously.

(a) 50 generations; (b)100 generations; (c) 200 generations; (d) 500 generations

Indeed, the optimal solution and standard deviation of $C R$ are stable while the generation increases to five hundred $\left(<10^{-4}\right)$. Therefore, We use the comparison matrix corresponding to the minimum $C R$ value of the five hundred generations evolutionary algorithm as the optimal comparison matrix to calculate the weight of each parameter. The calculation results are shown in Table 3.

Table 3 Weight of index layer

\begin{tabular}{|c|c|c|c|}
\hline Target Layer & Criterion Layer & Index Layer & Weight \\
\hline $\begin{array}{l}\text { Evaluation System of Geological Environment Carrying } \\
\text { Capacity of }\end{array}$ & $\begin{array}{l}\text { Geological } \\
\text { Environment }\end{array}$ & $\begin{array}{l}\text { Geological } \\
\text { Structure }\end{array}$ & 0.10676812 \\
\hline \multirow[t]{7}{*}{ Pingwu County } & & $\begin{array}{l}\text { Geological } \\
\text { Structure }\end{array}$ & 0.10782611 \\
\hline & & Topography & 0.10727444 \\
\hline & & Natural hazard & 0.10834189 \\
\hline & Ecosystem & Water Resources & 0.02623324 \\
\hline & & Mine Resources & 0.10817181 \\
\hline & Social Economy & Population & 0.21682408 \\
\hline & & Basic Facilities & 0.21856031 \\
\hline
\end{tabular}

\subsection{Results of Geological Eco-Environment Carrying Capacity in Pingwu County}


Based on the nine evaluation indexes in the three criteria of geological environment, ecological environment, and social environment, Figure 6 shows the results of GIS raster visualization. And then, we determinate the weight of each index by modified AHP method that used evolutionary algorithms. We calculated and superimposed multiple values of indicators through the GIS grid and finally obtained the geological environment carrying capacity map of Pingwu County.

(a) Lithology; (b) Topography; (c) Natural hazards; (d) Geological structure.

There are four indexes in geological environment criterion layer, as shown in Figure 6. Topography and geological structure contain two secondary factors, respectively. The geological structure mainly includes the influence of seismic faults, and it is quantified as fault distance and classification of ground shock. Similarly, Slope and elevation are select to represent the characteristics of topography. Other indexes can be expressed directly by a single factor which including natural hazards density and lithology.

According to the genetic type, material composition, structural characteristics, and physical and mechanical properties of the strata, the lithology is divided into five geological rock groups: clastic rock, carbonate rock, metamorphic rock, magmatic rock and pebble, which could be considered as hard rock, medium-hard rock, hard/soft interphase, soft rock and pebble soil,respectively. Topography index include slope range from to and elevation range from $600 \mathrm{~m}$ to $5440 \mathrm{~m}$, and they are divided into four levels. The main purpose of this study is to understand the geological carrying capacity after the Wenchuan earthquake, so the natural disaster index analyzes the disaster density distribution after the earthquake, mainly including debris flow, landslide, collapse and other disasters, and geological structure index includes fault distance and ground shock, which belong to quantitative indicators directly related to earthquake.

Figure 7 gives visualization results of two indexes in the ecological environment criterion layer, including water resources and mine resources. Water resources mainly come from the Fujiang River running through Pingwu County with a total length of $157 \mathrm{~km}$, which is the largest tributary of the Jialing River. Moreover, there are 15 Fujiang tributaries and 428 streams in the region, such as Qingyi River and Duobu River, the total river network density is up to $0.3 \mathrm{~km} / \mathrm{km}^{2}$. So it is a critical criterion of river distance for water resources. The study area is rich in iron, lead, zinc, manganese, gold, and other mineral resources. On the one hand, In the process of mine underground mining, mine roof caving, gushing water, precipitation induced groundwater level decline, part of the open-pit mining lead the natural slope instability eventually forms landslide. On the other hand, the arbitrary accumulation of abandoned soil and slag formed by mining provides more material sources for disasters such as collapse, landslide, and debris flow triggered by rainstorm and destroys the ecological environment.

The population density and basic facilities belong to the social economy criterion layer. Population density shows the degree of population aggregation in Pingwu County. As is shown in figure 7, the Population of Pingwu mainly concentrated in Longan Town, Crystal Town, Nanba Town, and radioactive reduction in population density centered on these areas. The population density of Tucheng Town and Xiangyan Town decreased sharply to below 50 people per square kilometer. The infrastructure in Pingwu County is mainly distributed along the river basin, in which Longan Town is the most concentrated. It is distributed linearly along the main river channel and tributaries of the Fujiang River. The infrastructure is mainly highway traffic, town construction, and ancillary facilities.

Indeed, we use the weight of each index (Table 3) obtained by the optimal comparison matrix and implement grid superposition to draw the geological eco-environment carrying capacity map of Pingwu County.

The carrying capacity of the eco-geological environment in Pingwu County involves four categories, there are high, medium, low, and very low, respectively. Furthermore, these categories can be divided into two states: equilibrium (critical overload) and surplus (non-overload). The equilibrium state includes high and medium carrying capacity, which means the geological disasters in the region are relatively developed, and the development area is limited. Moreover, both low and very low carrying capacity all belong to the surplus state, and the areas of this state are usually less developed with low population

Page $7 / 18$ 
density. As can be seen in Figure 8, high carrying capacity areas mainly located along the Fujiang River and both sides of the Pingtong River, including slope sections on both sides of the valley and human activities to the buffer zone of nature reserves, as well as some low-mountain and hilly landform areas. The critical overload capacity in these areas mainly due to frequent geological disasters and human activities. Similarly, The areas in Pingwu County with low population density and fewer geological disasters belong to the surplus state, mainly including nature reserves in Baima Town, Tucheng Town, and Huya Town.

\section{Discussion}

Pingwu County is located in the upper reaches of the Yangtze River which regional geological structure is complex. Especially after the Wenchuan earthquake, the unique geological structure, earthquake, debris flow, landslide, and other natural hazards in the coastal area become a significant barrier to the recovery and development of cities and towns(Song et al. 2016; Ping and Li 1994).

The multi-index comprehensive evaluation method of geological environment carrying capacity can be used to evaluate geological conditions in Pingwu quantitatively. Analytic Hierarchy Process(AHP) is the standard multi-index quantitative analysis method. However, the expert scoring of AHP is greatly influenced by subjective factors, so we combine fuzzy scoring matrix and evolutionary algorithms to find the comparison matrix with the minimum consistency ratio.

According to previous studies about evaluating environment carrying capacity by AHP(Dai, Lee, Zhang 2001; Liu and Li 2020; Bozdağ, Yavuz, Dönertaş 2016), most of consistency ratio $C R$ in these researches are range from 0.01 to 0.07 . As shown in Fig. 9, compared to the result of modified AHP that obtain consistency ratio $C R<10^{-4}$, other results of $C R$ are much larger than it, these judgment matrices are not consistent enough, even though they all pass the consistency checking. As a result, the modified AHP can evaluate multi-index quantification problems more objectively and reasonably than traditional ways.

The results of eco-geological carrying capacity in Pingwu provide a reference for post-earthquake reconstruction and ecological restoration in the future. As can be seen in Fig. 10, high carrying capacity areas accounted for $22.64 \%$ of the total area of Pingwu, mainly located along the Fujiang River and both sides of the Pingtong River. The advantages of these areas are convenient transportation, rich water and soil resources, and multiple town gathering places. Moreover, the disadvantages include that these areas belong to the Zhongshan landform structure, so there are a lot of geological disasters that threatened the safety of towns and highways, and the development of this area is limited in the future. Furthermore, it is suggested that these areas should strengthen the combination of engineering and ecological management measures to carry out ecological slope management measures, which are beautiful and effective. The reconstruction plan should pay more attention to optimize the design of critical towns, give full adapted use to the characteristics of the geological environment of the towns.

The medium carrying capacity area accounts for $33.48 \%$ of the total area, primarily located on both sides of the valley and the transition area from human activities to nature reserves. The area belongs to hilly landform zone, and there are plenty of mineral resources and fewer human engineering activities in this area. However, it is hard to implement the restoration of the mine area and the treatment of aggregation of local small geological disasters. The planning proposal of this region focuses on the scientific and rational exploitation of stock mines. For instance, the environmental restoration of abandoned mining areas, the control of secondary disasters caused by mining, and the reclamation of mining areas.

The areas with low and very low eco-geological environmental carrying capacity account for $32.64 \%$ and $11.24 \%$ of the total area, respectively, which are both located in nature reserves, with less human engineering activities and low geological disaster risk. It is necessary to optimize the construction of the center in the region, control the scale of regional construction, and maintain the original nature of ecological species in nature reserves. 


\section{Conclusion}

In view of the severe threat of earthquakes and geological disasters to the towns in mountainous areas of southwest China, and the characteristics that the urban construction is deeply restricted by the geological environment conditions and the susceptibility of geological disasters, this paper selects Pingwu County, a key town in the typical and representative Fu River Basin, as a study area. Based on many field investigations and collected data in Pingwu, we conduct a scientific evaluation of the eco-geological environment carrying capacity of the towns by modified AHP with evolutionary algorithms. It provides a scientific basis for the long-term planning and development of Pingwu County after the Wenchuan earthquake. The result in detail as follows: (1) The traditional AHP is modified by evolutionary algorithms. The fuzzy scoring matrix is used to determine the optimal weight coefficient of consistency, which avoids difficulties of consistency testing and the influence of subjective factors brought by expert scoring. (2) The modified AHP and spatial processing technology support the evaluation of geological environment carrying capacity. The results show that this method is reasonable. However, due to the effect of strong geological tectonic motion, the evaluation system is only applicable to similar areas of cities and towns in southwestern mountainous areas. (3) According to the classification results of eco-geological environmental carrying capacity, the advantages and disadvantages of different regions in the planning process are given, and specific suggestions are given for the future planning of different regions.

\section{Declarations}

\section{Acknowledgements}

The authors would like to thank the reviewers for their valuable suggestions and comments on the Ph.D. Qiang Su(Nanjing University) about data visualization.

\section{Funding}

This research was funded by National Natural Science Foundation of China (41804089).

\section{Conflicts of Interest,}

The authors declare no conflict of interest.

\section{Availability of data and material}

The data that support the findings of this study are available from the corresponding author, $t$ Yimin Liu, upon reasonable request.

\section{Code availability}

The code of modified AHP can be acquired in Supplement Modified_AHP.py.

\section{Authors' contributions}

The authors equally contribute to this manuscript.

\section{Ethics approval}

The authors declare no conflict of interest.

\section{References}

Bishop, A B (1974) Carrying capacity in regional environmental management (US Government Printing Office). 
Bozdağ, A, F Yavuz,A Dönertaş (2016) AHP and GIS based land suitability analysis for Cihanbeyli (Turkey)

County. Environmental Earth Sciences 75: 813. https://doi.org/ 10.1007/s12665-016-5558-9

Chen, Q, L Z Yi,H L Shi (2004) Thoughts and Basic Methods of Regional Geological Hazard Risk Assessment, Journal of Geomechanics 10: 71-80. https://doi.org/10.1016/S0960-0779(03)00420-X

Cherp, A (2001) Environmental assessment in countries in transition: evolution in a changing context, J Environ Manage 62: 357-74. https://doi.org/10.1006/jema.2001.0438

Dai, F C, C F Lee,X H Zhang (2001) GIS-based geo-environmental evaluation for urban land-use planning: a case study, Eng Geol 61: 257-71. https://doi.org/10.1016/S0013-7952(01)00028-X

Liu, R, C Wang, J Hao, S U Baolin,M A Yongliang (2009) Measuring Environmental Carrying Capacity, Journal of Basic Science and Engineering 17: 49-61. https://doi.org/ 10.16058/j.issn.1005 -0930.2009.01.003

Liu, Y,L Li (2020) Mountainous City Featured Landscape Planning Based on GIS-AHP Analytical Method, ISPRS International Journal of Geo-Information 9: 211. https://doi.org/ 10.3390/ijgi9040211

Ping, Y,Y Li (1994) Geologic Tectonic and Disasters in Alongshore Area at the Upper Reaches of the Yantgze River and the Influences on Urban Development, Journal of Southwest China Institute of Technology 9(2): 34-41.

https://doi.org/CNKI:SUN:XNGX.0.1994-02-006

Qin, X W, Z Zhang, J J Yang,T Huang (2009) Analysis on the Secondary Geological Disaster in Pingwu of 5.12 Wenchuan Earthquake,Sichuan Based on Remote Sensing Information, Geological Science and Technology Information 28: 12-15. https://doi.org/ 10.1016/S1003-6326(09)60084-4

Rees, W E (1992) Ecological Footprints and Appropriated Carrying Capacity: What Urban Economics Leaves Out, Environ Urban 4: 121-30. https://doi.org/10.1177/095624789200400212

Saaty, T L (2008) Decision making with the analytic hierarchy process, International Journal of Services Sciences 1: 83-98. https://doi.org/10.1504/IJSSCI.2008.017590

Saaty, T L,K P Kearns (1985) The Analytic Hierarchy Process.Analytical Planning.

Saaty, T L,L G Vargas (2012) Models, methods, concepts and applications of the analytic hierarchy process. Boston: Kluwer Academic Pub-lishers.

Song, Z, N I Hua-Yong, H F Zhou,W Feng (2016) Risk Assessment of Seismic Landslide within Small Region Based on MultiLevel Physical and Mechanical Parameters: A Case Study of Shimian and Adjacent Areas in the Upper Reaches of Yangtze River, Journal of Geomechanics 22: 760-70. https://doi.org/CNKI:SUN:DZLX.0.2016-03-029

Trevisan, M, L Padovani,E Capri (2000) Nonpoint-source agricultural hazard index: a case study of the province of cremona, Italy, Environ Manage 26: 577-84. https://doi.org/ 10.1007/s002670010114

Vatn, A (2009) An institutional analysis of methods for environmental appraisal, Ecol Econ 68: 2207-15. https://doi.org/ 10.1016/j.ecolecon.2009.04.005

Wang, K F,L I Na (2015) Evaluation of Geo-environmental Carrying Capacity in Shandong Peninsula Based on AHP and GIS Coupling Model, China Population,Resources and Environment 25: 224-27. https://doi.org/CNKI:SUN:ZGRZ.0.2015-S1-057

Wang, X, K Masaki,K Irikura (2011) Building Damage Criteria from Strong Ground Motion Characteristics during the 2008 Wenchuan Earthquake, Journal of Earthquake Engineering 15: 1117-37. https://doi.org/10.1080/13632469.2011.552311 
Wang, Z,F C Yi. (2009) AHP-based evaluation of occurrance easiness of geological disasters in Mianyang City, Journal of Natural Disasters 18: 14-23. https://doi.org/ 10.1002/9780470611807.ch2

Ye, S, G Zhai,J Hu (2011) Damages and Lessons from the Wenchuan Earthquake in China, Human and Ecological Risk Assessment: An International Journal 17: 598-612. https://doi.org/ 10.1080/10807039.2011.571086

Zhang, W J, J Y Lin, J A Peng,Q F Lu (2010) Estimating Wenchuan Earthquake induced landslides based on remote sensing, International Journal of Remote Sensing 31: 3495-508. https://doi.org/ 10.1080/01431161003727630

Zhao J,Dan Q (2003) Mathematical Modeling and Mathematical Experiment. Beijing: Higher Education Press.

Zhu, Y (2009) Empirical Analysis on Environmental Carrying Capacity in the Guangxi Beibu Gulf Economic Region, Around Southeast Asia 9: 87-91.

\section{Figures}

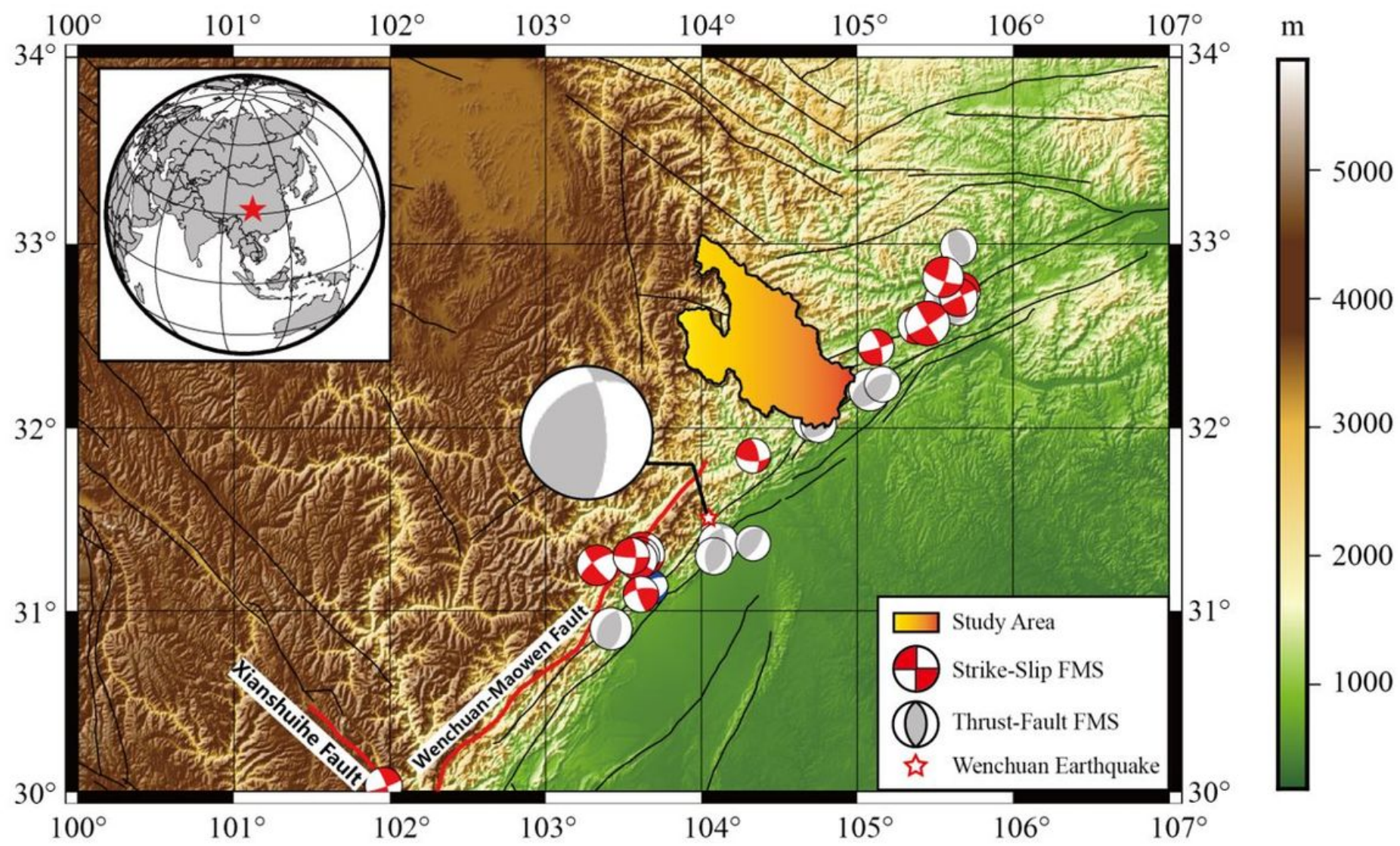

Figure 1

Location of Pingwu country and the earthquake distribution in 2008 


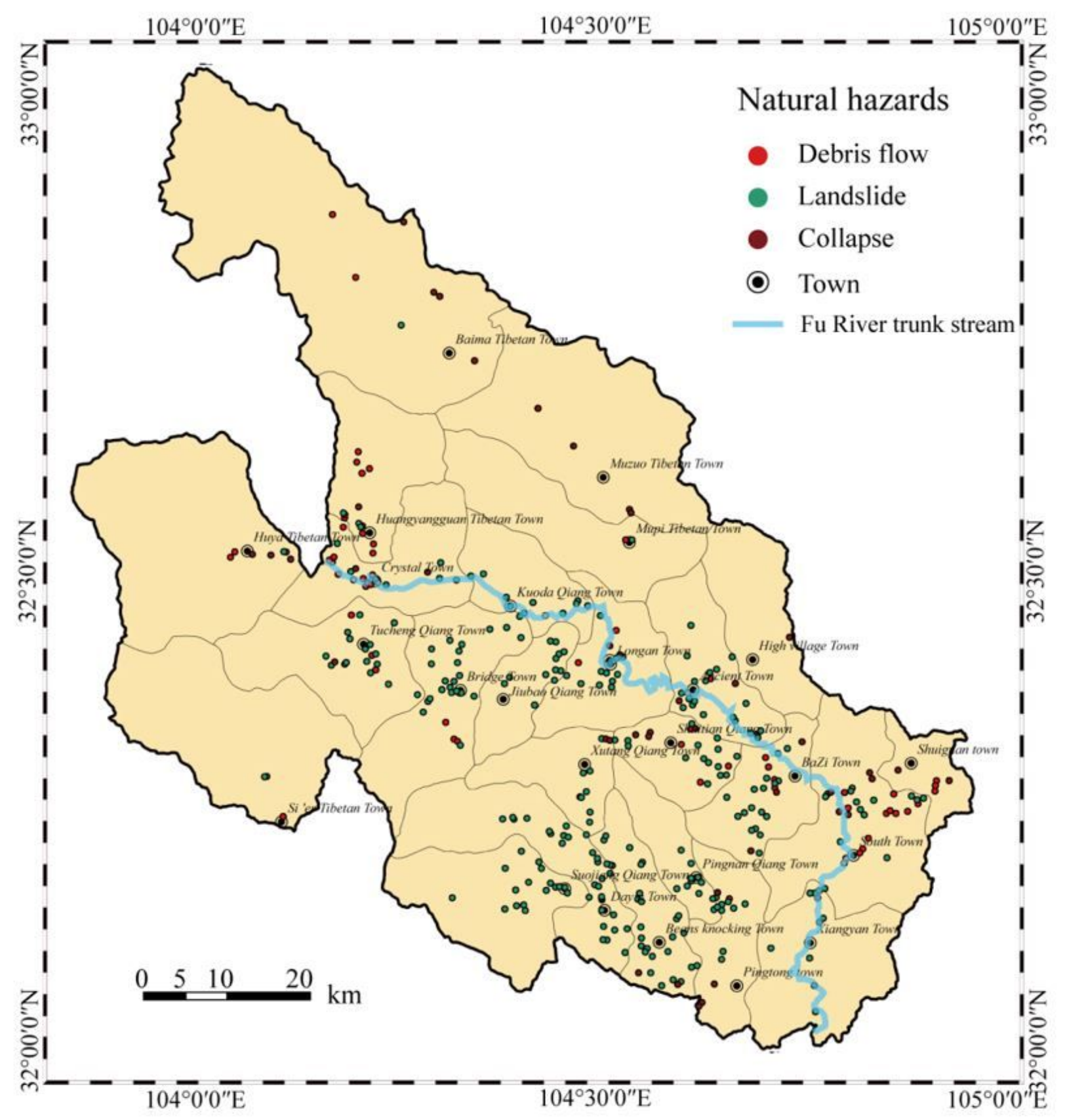

Figure 2

Location of natural hazards in Pingwu country

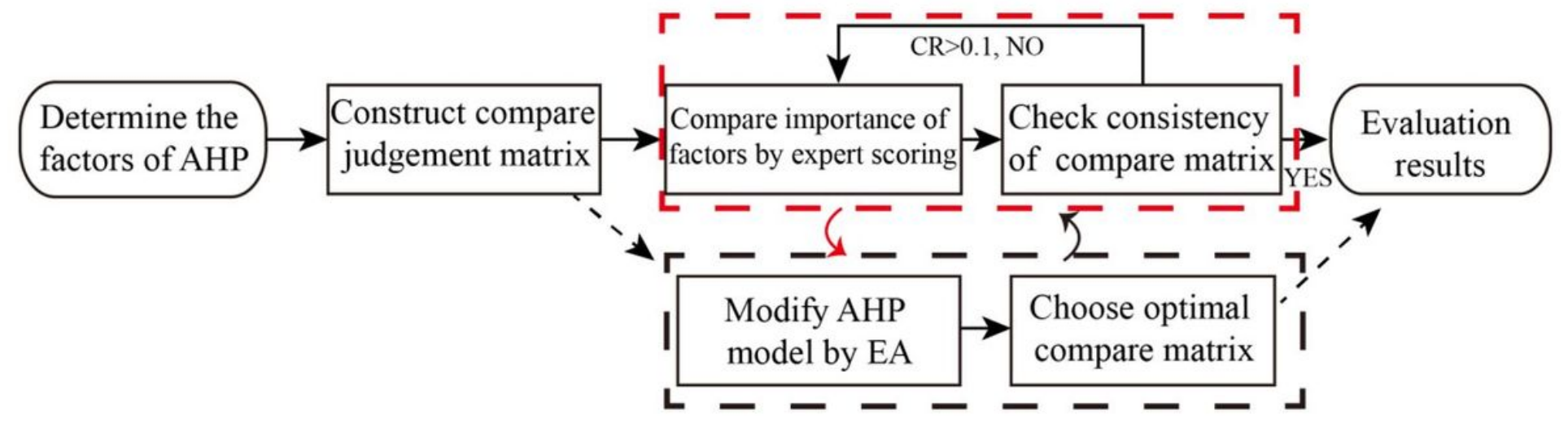


Figure 3

The process of modified AHP

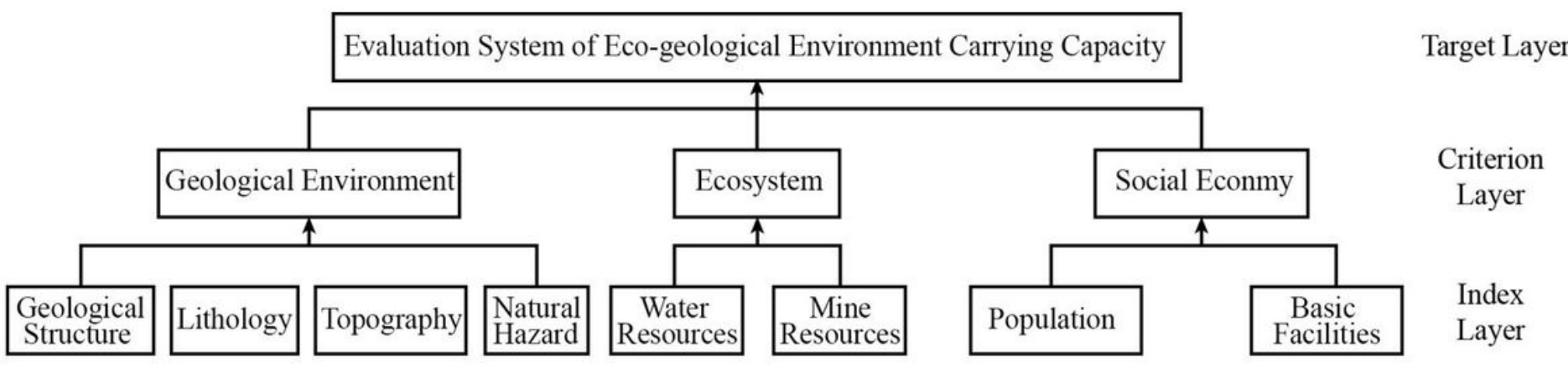

\section{Figure 4}

Evaluation index system structure of geological eco-environment carrying capacity in Pingwu county
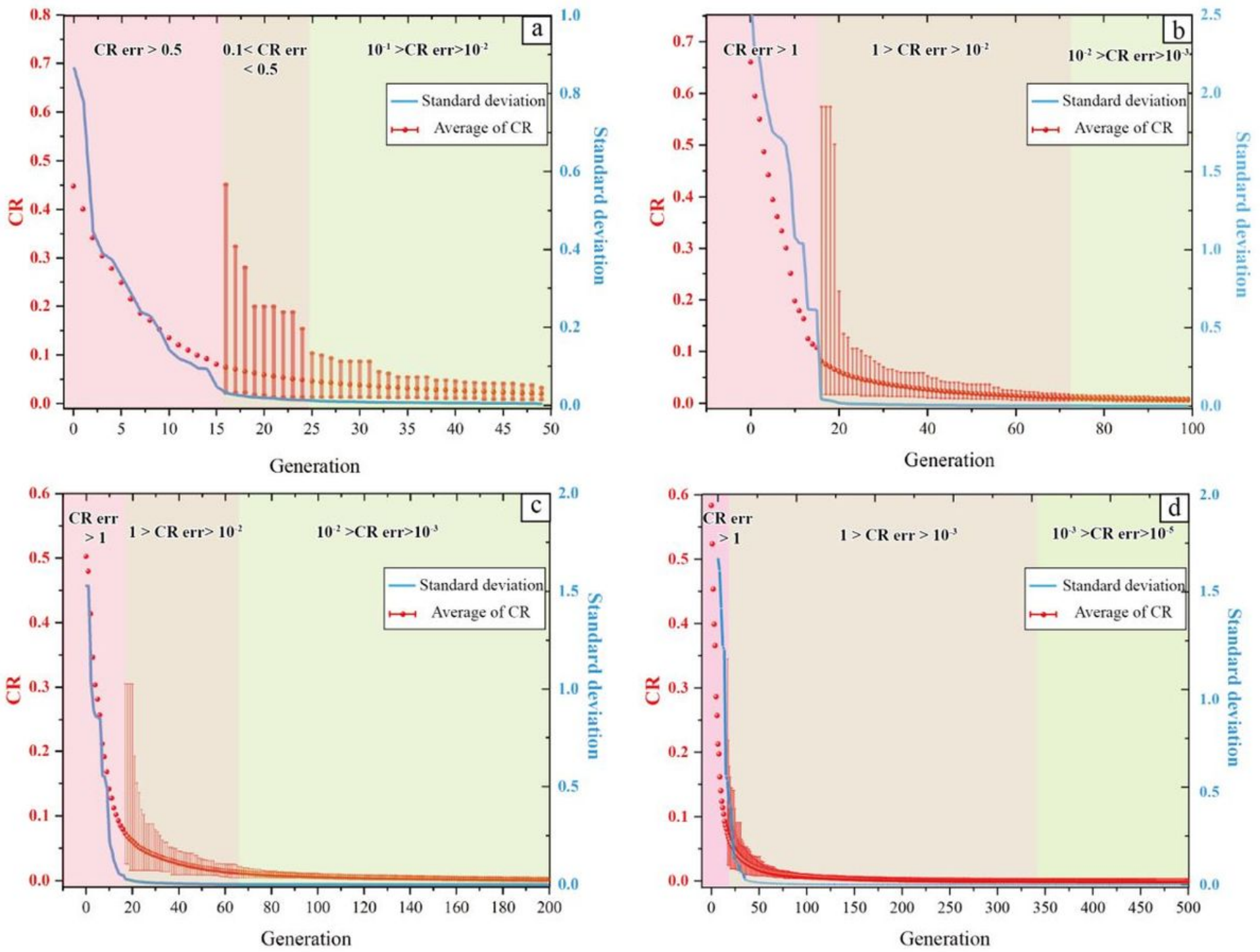

Figure 5

Process of evolutionary algorithms in different evolution generations. 


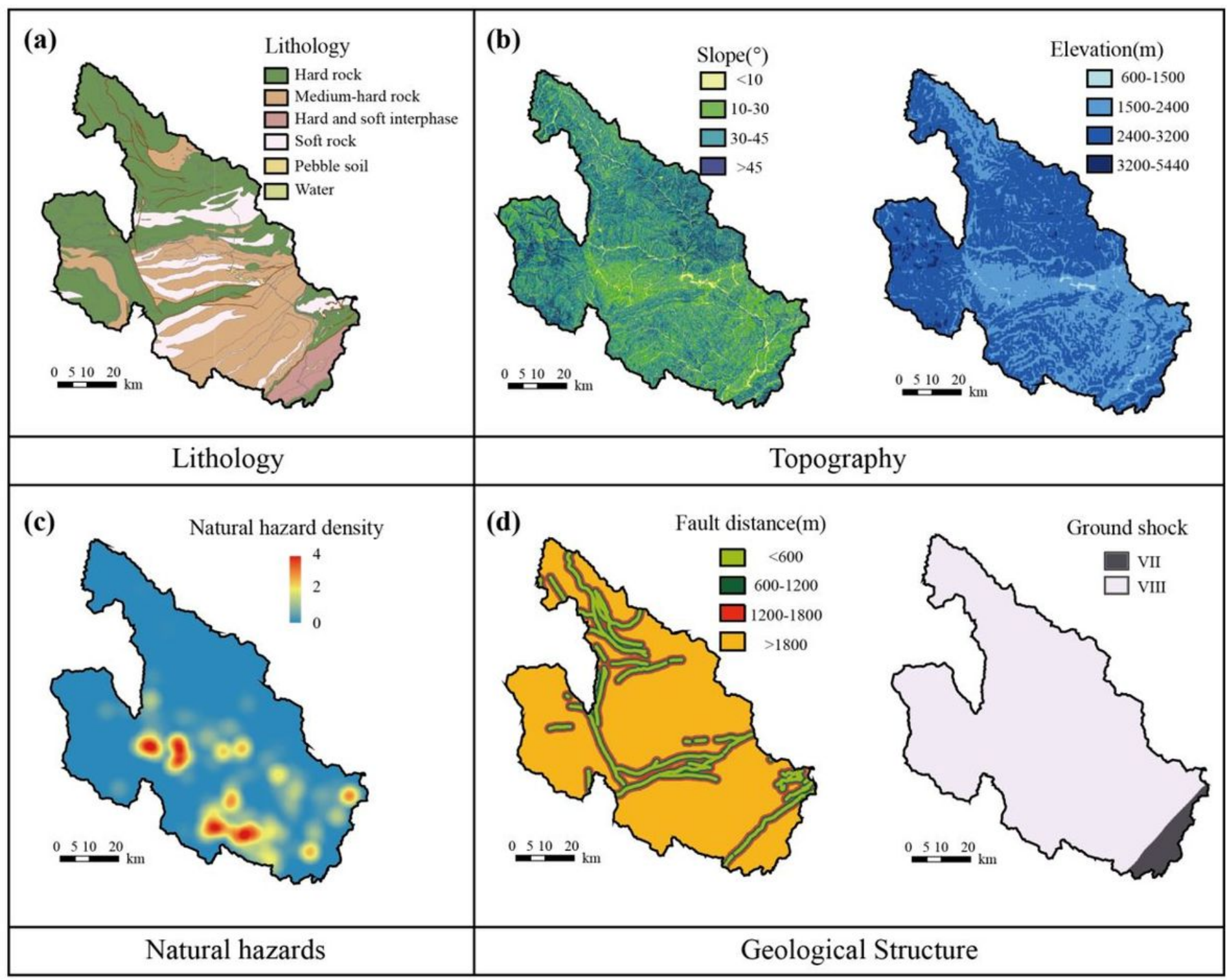

Figure 6

Visualization result of indexes in geological environment criterion layer. 


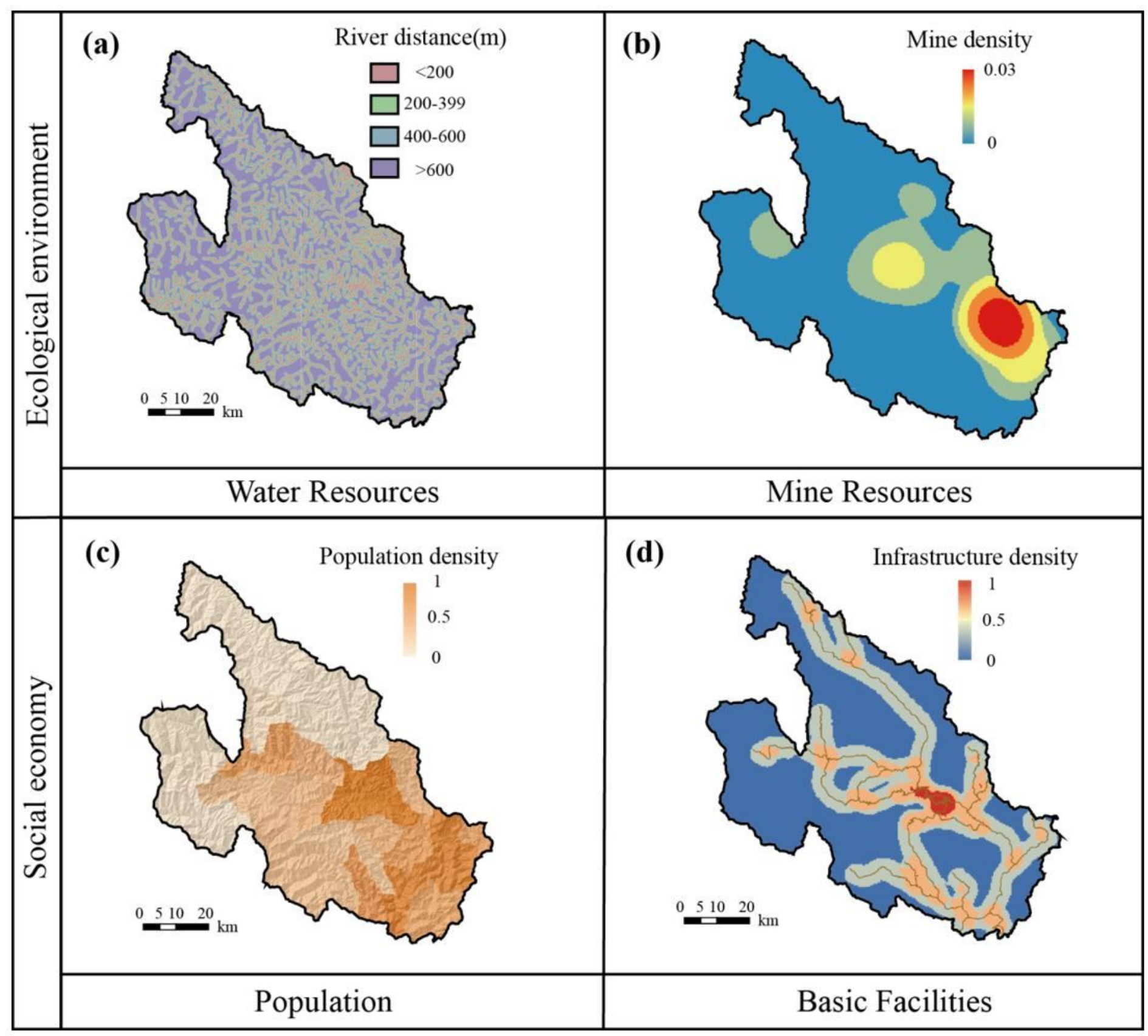

Figure 7

Visualization result of factors in the ecological environment and social economy criterion layer 


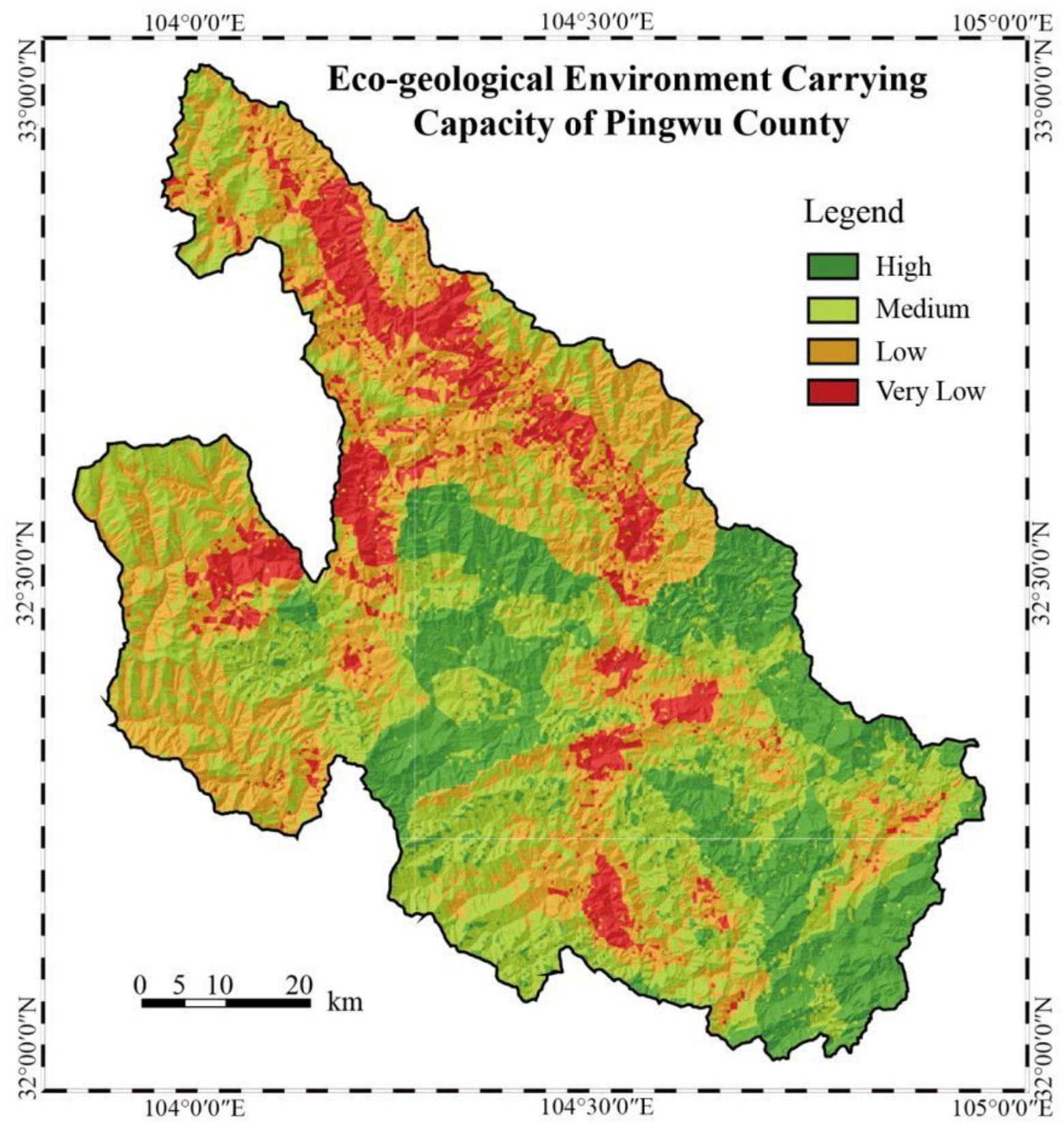

Figure 8

Classification Map of Eco-geological Environment Carrying Capacity in Pingwu County 


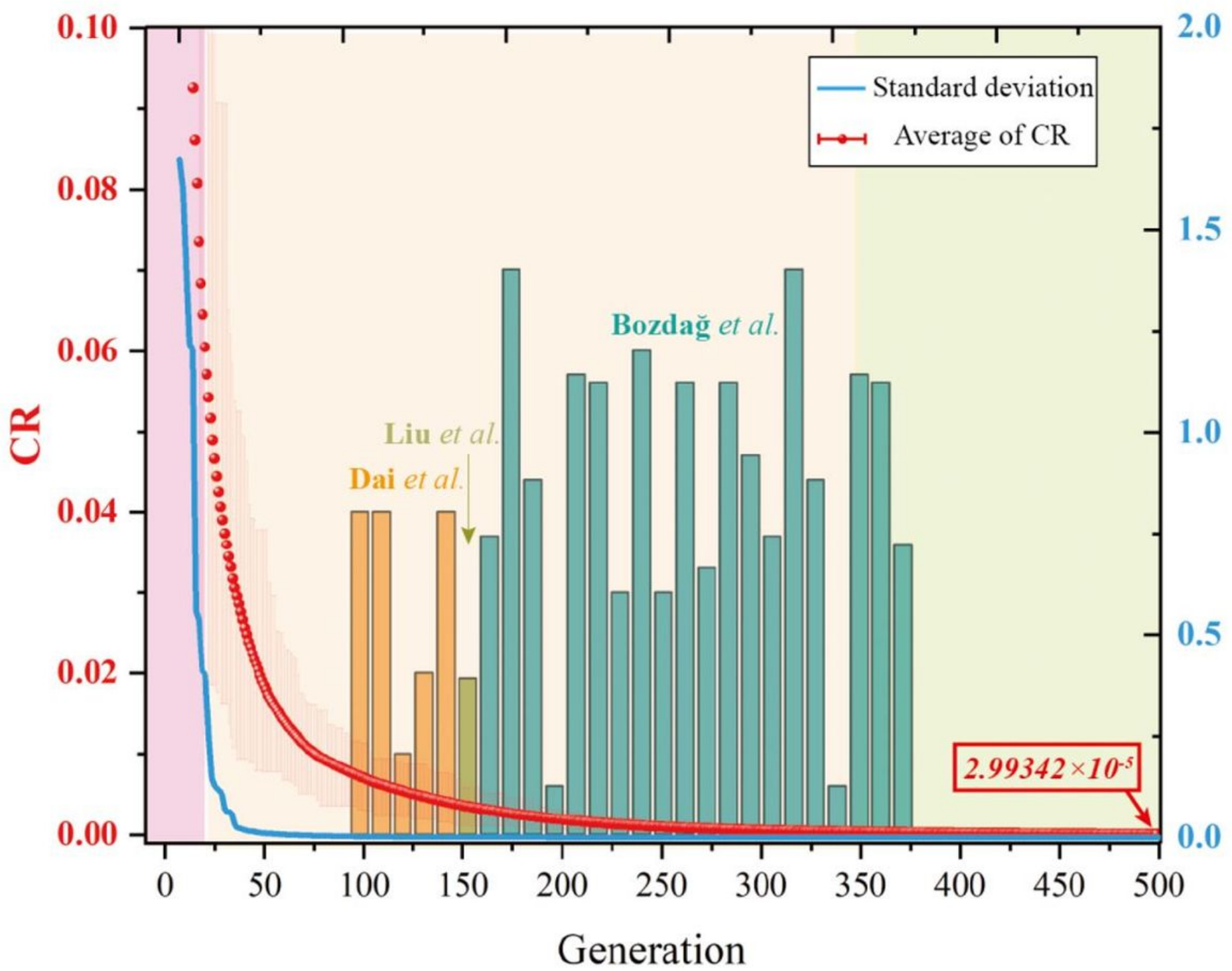

Figure 9

Consistency ratio of traditional and modified AHP(Dai et al. 2001; Liu and Li 2020; Bozdağ et al. 2016) 


\section{Area Ratio of Eco-geological Environment Carrying Capacity in Pingwu}

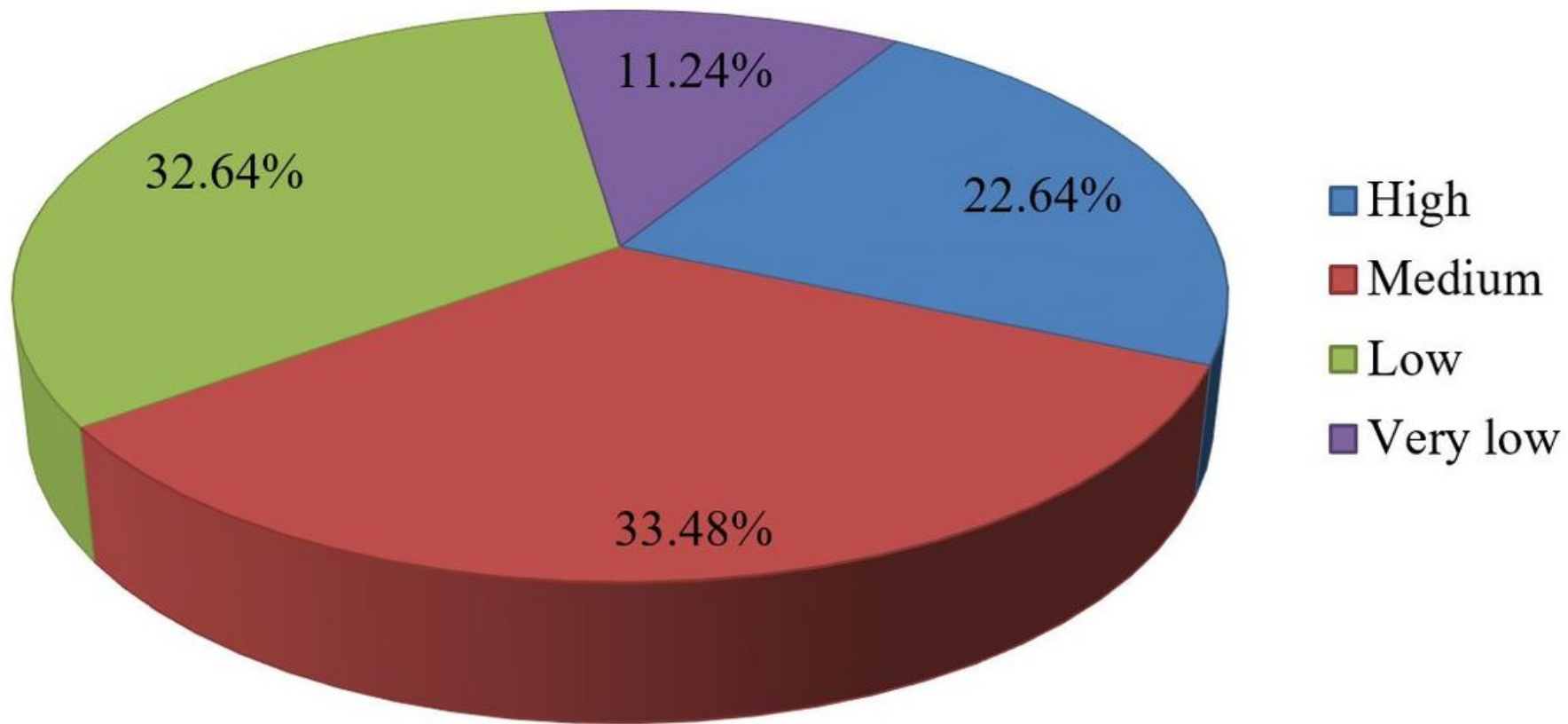

Figure 10

The area ratio of Eco-geological Environment Carrying Capacity in Pingwu County

\section{Supplementary Files}

This is a list of supplementary files associated with this preprint. Click to download.

- ModifiedAHP.py 